\author{
GAME THEORY AND \\ MATHEMATICAL ECONOMICS \\ BANACH CENTER PUBLICATIONS, VOLUME 71 \\ INSTITUTE OF MATHEMATICS \\ POLISH ACADEMY OF SCIENCES \\ WARSZAWA 2006
}

\title{
SOLIDARITY AND COOPERATIVE BARGAINING SOLUTIONS
}

\author{
NAOKI YOSHIHARA \\ Institute of Economic Research, Hitotsubashi University \\ Naka 2-1, Kunitachi, Tokyo 186-8603, Japan \\ E-mail: yosihara@ier.hit-u.ac.jp
}

\begin{abstract}
In this paper, we consider production economies with possibly unequal production skills and with the possibility of technological innovations, in which resource allocations are determined via bargaining among individuals. We define the Nash (resp. the Kalai-Smorodinsky) bargaining solution as the (bargaining) allocation rule whose utility outcomes just result in the Nash (resp. the Kalai-Smorodinsky) bargaining outcomes. Two new axioms regarding compensation for low skill agents are introduced as weak versions of the solidarity condition w.r.t. change in production skills. Then, we show that the Nash (resp. the Kalai-Smorodinsky) bargaining solution is the unique Efficient and Symmetric bargaining allocation rule satisfying both the responsibility and one of the compensation requirements.
\end{abstract}

1. Introduction. In this paper we consider resource allocation problems in production economies with possibly unequal skills, as well as with variable commodities, in which the change in the types of produced commodities is due to the change in production technology. Assuming that the resource allocation is determined via bargaining among individuals, we axiomatically characterize bargaining solutions in those economies. In contrast to the classical bargaining theory originating with Nash (1950), we focus on allocation rules, each of which maps each economy to a subset of feasible allocations whose utility values are just the bargaining outcomes. In this way, we adopt the axioms which refer explicitly to concrete data on underlying economic environments, rather than just to the geometric data of utility possibility sets.

2000 Mathematics Subject Classification: Primary 91A.

Journal of Economic Literature Classification: C71, C78, D63, D71.

Key words and phrases: Nash bargaining solution, Kalai-Smorodinsky solution, responsibility and compensation, solidarity w.r.t. change in production skills.

The author is specially thankful to the referee whose advice drastically improved the readability of this paper.

The paper is in final form and no version of it will be published elsewhere. 
Such an approach is useful to make clear non-welfaristic properties of bargaining solutions beyond the welfaristic discussions in the Nash-type classical approach. For example, in our setting of production economies, this approach may make it possible to discuss the important issues of whether and/or how each of the following should influence the bargaining outcome; the inequality of individuals' labor skills, the individuals' developments of "expensive tastes" [Dworkin (1981a,b)] for which they should be responsible, and the effect of technological innovation. Such issues disappear in the classical approach, because of its implicit imposition of the axiom of Welfarism [Roemer (1988)] which requires solutions to assign the same utility allocation to all the economies giving rise to the same utility possibility sets.

Our model is relevant to bargaining problems over the social compensation for low skills. Regarding the problem of social compensation for low skills, Dworkin (1981b) developed the theory of equality of resources, which says that the relatively less-preferred situation of an individual should be compensated by the society if his/her situation is due to his/her low skill, but not if it is caused by his/her type of utility function such as "expensive taste." This is because, as Dworkin (1981b) and Fluerbaey and Maniquet (1996, 1999) discussed, an individual should be responsible for his/her less-preferred situation if such a situation is caused by his/her free will which is represented by his/her own utility function, while such an argument may not be applied if his/her situation is due to his/her low skill.

Motivated by such a viewpoint of responsibility and compensation [Fluerbaey and Maniquet $(1996,1999)$ ], we provide new axiomatic characterizations of the Nash [Nash (1950)] solution and the Kalai-Smorodinsky [Kalai and Smorodinsky (1975)] solution. Through the characterizations, we may obtain a new insight on the property of these solutions in terms of responsibility and compensation. One axiom can be interpreted as being relevant to responsibility for utility functions, and the other three axioms as being relevant to compensation for low skills. The responsibility axiom is Independence of Utility Intensities (IUI), while the compensation axioms we are interested in here are Skill Monotonicity (SM) and its weaker versions, $\alpha$-Weak Skill Monotonicity ( $\alpha \mathbf{W S M )}$ and $\beta$-Weak Skill Monotonicity ( $\beta$ WSM).

In the bargaining problem over compensation for low skills, the most desirable solution would satisfy both the responsibility axiom IUI and the compensation axiom SM. Unfortunately, this was proven impossible by Yoshihara (2003), thus, we should pursue the second best solution instead. This paper will discuss the Nash solution as a plausible second best solution, in the sense that it is the unique Efficient and Symmetric bargaining solution satisfying both IUI and $\alpha$ WSM. Moreover, $\alpha$ WSM together with the Pareto Efficiency axiom have an appealing content as a solidarity condition w.r.t. someone's worsening of labor skills. We also show that the Kalai-Smorodinsky solution is the unique Efficient and Symmetric bargaining solution satisfying both IUI and $\beta$ WSM. Since $\beta$ WSM seems to be not as ethically appealing as $\alpha \mathbf{W S M}$, our main results may indicate that the Nash solution is most appropriate for the bargaining problem over compensation for low skills, whenever we take into account responsibility and compensation as essential principles. 
In the following discussion, section 2 defines a basic model of economies, allocation rules, and bargaining solutions. Section 3 introduces the axioms on allocation rules. Section 4 provides our main results. For the sake of expositional convenience, all the involved proofs are relegated into Section 5 .

2. Model. As explained in section 1, we look at resource allocations in production economies with possibly unequal skills, and with variable commodities. To treat the case of variable commodities, we start from introducing (possibly) infinitely many types of commodities. The universe of such "potential commodities" is denoted by $C$, and the class of non-empty and finite subsets of $C$ is designated by $\mathcal{M}$, with generic elements, $K, L, M, \ldots$ The cardinality of $M \in \mathcal{M}$ is denoted by $\# M$. Given $M \in \mathcal{M}$, let $\mathbb{R}_{+}^{m}$, where $m=\# M$, designate the Cartesian product of \#M copies of $\mathbb{R}_{+}$indexed by the numbers of $M$, where $\mathbb{R}_{+}$denotes the set of non-negative real numbers. There is also one type of labor input, which is denoted by $x \in \mathbb{R}_{+}$, to be used to produce any potential commodity.

Each production technology is represented by a set of the input-output vectors of labor and some finite types of commodities. Thus, for any finite types of commodities $M \in \mathcal{M}$, one technology that can produce up to $M$-goods is described by a production possibility set $Y \subseteq \mathbb{R}_{+} \times \mathbb{R}_{+}^{m}$, where it is assumed that:

A.1 $0 \in Y$.

A.2 $Y$ is closed, convex, and comprehensive.

A.3 $\exists t=(x, y) \in Y$ such that $\exists$ a commodity $f \in M$ s.t. $y_{f}>0$.

The above A.1, A.2, and A.3 are standard assumptions for production possibility sets in economics. The universal set of such production possibility sets which produce up to $M$-goods is denoted by $\mathcal{Y}^{M}$. Let $\mathcal{Y} \equiv \bigcup_{M \in \mathcal{M}} \mathcal{Y}^{M}$. Let $\partial Y \equiv\left\{(x, y) \in Y \mid \nexists\left(x^{\prime}, y^{\prime}\right) \in Y\right.$ s.t. $\left.\left(-x^{\prime}, y^{\prime}\right) \gg(-x, y)\right\}$. $^{1}$

By assuming one type of labor, it follows that every individual's labor is homogeneous, although their labor skills are possibly unequal. The population in the economy is given by the set $N=\{1, \ldots, n\}$, where $2 \leq n<+\infty$. Assume that all individuals have the same upper bound of labor time $\bar{x}, 0<\bar{x}<+\infty$. Each individual $i$ is characterized by a labor skill which is represented by a non-negative real number, $s_{i} \in \mathbb{R}_{+}$. The number $s_{i}$ indicates $i$ 's labor supply per hour measured in efficiency units. Thus, if $x_{i}$ is the labor time expended by $i$, then his labor supply in efficiency units is $s_{i} x_{i}$. Let us denote the universal set of labor skills for all individuals by $\mathcal{S} \subseteq \mathbb{R}_{+}$.

Each individual is also characterized by his own utility function defined over a consumption space. The consumption space is given by $[0, \bar{x}] \times \mathbb{R}_{+}^{m}$ whenever there exist $m$ types of commodities that are able to be produced in the society. Let $\mathcal{U}^{M}$ be the set of all (real-valued) concave and continuous utility functions defined on $[0, \bar{x}] \times \mathbb{R}_{+}^{m}$, such that any $u \in \mathcal{U}^{M}$ is non-increasing in $[0, \bar{x}]$, non-decreasing in $\mathbb{R}_{+}^{m}, u(0, \mathbf{0})=u(\bar{x}, \mathbf{0})=0$, and

\footnotetext{
${ }^{1}$ For any two vectors $\mathbf{a}=\left(a_{1}, \ldots, a_{p}\right)$ and $\mathbf{b}=\left(b_{1}, \ldots, b_{p}\right), \mathbf{a} \geq \mathbf{b}$ if and only if $a_{i} \geq b_{i}$ $(i=1, \ldots, p), \mathbf{a}>\mathbf{b}$ if and only if $\mathbf{a} \geq \mathbf{b}$ and not $(\mathbf{b} \geq \mathbf{a})$, and $\mathbf{a} \gg \mathbf{b}$ if and only if $a_{i}>b_{i}$ $(i=1, \ldots, p)$.
} 
for all $(x, y) \in[0, \bar{x}] \times \mathbb{R}_{+}^{m}, \lim _{t \rightarrow \infty}(1 / t) \cdot u(x, t y)=0$. Those assumptions on utility functions are also standard in economics. Note that each utility function is defined dependent on the type of commodities, which the society has the technology to produce.

Given $M \in \mathcal{M}$, an economy with $M$-commodities is described by a list $\mathbf{e}=(M, \mathbf{u}, \mathbf{s}, Y)$ $=\left(M,\left(u_{i}\right)_{i \in N},\left(s_{i}\right)_{i \in N}, Y\right)$, where $M \in \mathcal{M}, \mathbf{u} \in \mathcal{U}^{M n}, \mathbf{s} \in \mathcal{S}^{n}, Y \in \mathcal{Y}^{M}$, and $\mathcal{U}^{M n}$ and $\mathcal{S}^{n}$ stand, respectively, for the $n$-fold Cartesian product of $\mathcal{U}^{M}$ and that of $\mathcal{S} .^{2}$ Let $\mathcal{E}^{M}$ be the class of all such economies with $M$-goods. Let $\mathcal{E} \equiv \bigcup_{M \in \mathcal{M}} \mathcal{E}^{M}$. Note that this definition of economies implies that the change in the dimension of consumption space is caused by the change in production technology, while it may also induce the change of individuals' utility functions as discussed above. For instance, if a new commodity is "invented" or "discovered" through a technological innovation, then the dimension of consumption spaces of all individuals becomes higher, which may also drastically change the profile of all individuals' utility functions in the society. This kind of situation is worth discussing when we consider bargaining problems. For instance, the issue of how the changes in economic environments due to the technological innovation should or should not influence the consequences of bargaining between employees and employers might be interesting, and our model of economies can treat such an issue.

Given $\mathbf{e}=(M, \mathbf{u}, \mathbf{s}, Y) \in \mathcal{E}^{M}$, a vector $\mathbf{z}=\left(z_{i}\right)_{i \in N} \in\left([0, \bar{x}] \times \mathbb{R}_{+}^{m}\right)^{n}$ constitutes a feasible allocation for $\mathbf{e} \in \mathcal{E}^{M}$ if for all $i \in N, z_{i}=\left(x_{i}, y_{i}\right)$, and $\left(\sum s_{i} x_{i}, \sum y_{i}\right) \in Y$. Since the profiles of labor time and of skills are respectively $\left(x_{i}\right)_{i \in N}$ and $\left(s_{i}\right)_{i \in N}$, the aggregate amount of labor input in efficiency units is $\sum s_{i} x_{i}$, which is transformed into $M$-commodities through the production possibility set $Y$. We denote by $Z(\mathbf{e})$ the set of feasible allocations for $\mathbf{e} \in \mathcal{E}^{M}$. Let $Z(\mathcal{E}) \equiv \bigcup_{\mathbf{e} \in \mathcal{E}} Z(\mathbf{e})$.

Given $\mathbf{e}=(M, \mathbf{u}, \mathbf{s}, Y) \in \mathcal{E}$ and $\mathbf{z} \in Z(\mathbf{e}), \mathbf{z}$ is a Pareto efficient (resp. weakly Pareto efficient) allocation for $\mathbf{e}$ if there is no $\mathbf{z}^{\prime} \in Z(\mathbf{e})$ such that $u_{i}\left(z_{i}^{\prime}\right) \geq u_{i}\left(z_{i}\right)$ for all $i \in N$, and $u_{j}\left(z_{j}^{\prime}\right)>u_{j}\left(z_{j}\right)$ for some $j \in N$ (resp. $u_{i}\left(z_{i}^{\prime}\right)>u_{i}\left(z_{i}\right)$ for all $\left.i \in N\right)$. Denote the set of Pareto efficient (resp. weakly Pareto efficient) allocations for e by $P E(\mathbf{e})$ (resp. $W P E(\mathbf{e}))$. Given $\mathbf{e}=(M, \mathbf{u}, \mathbf{s}, Y) \in \mathcal{E}$, the utility possibility set of $\mathbf{e} \in \mathcal{E}$ is:

$$
S(\mathbf{e}) \equiv\left\{\overline{\mathbf{u}}=\left(\bar{u}_{i}\right)_{i \in N} \in \mathbb{R}_{+}^{n} \mid \exists \mathbf{z}=\left(z_{i}\right)_{i \in N} \in Z(\mathbf{e}), \forall i \in N, \bar{u}_{i}=u_{i}\left(z_{i}\right)\right\} .
$$

Note that the utility possibility set $S(\mathbf{e})$ is a compact, comprehensive, convex set in $\mathbb{R}_{+}^{n}$ containing the origin. Let $\Sigma \equiv\left\{S \subseteq \mathbb{R}_{+}^{n} \mid \exists \mathbf{e} \in \mathcal{E}, S=S(\mathbf{e})\right\}$ be the class of all such utility possibility sets.

Let $d=\mathbf{0} \in \mathbb{R}_{+}^{n}$ denote the disagreement point in this society. We identify a pair of the utility possibility set, $S$, and the disagreement point $d$ as a bargaining game. Then, a bargaining solution is a function $F: \Sigma \times\{d\} \rightarrow \mathbb{R}_{+}^{n}$ such that for every $S \in \Sigma, F(S, d) \in S$. Since $d=\mathbf{0}$ by the assumption of $u_{i}(0, \mathbf{0})=0$ for all $i \in N$, we write only $F(S)$ instead of $F(S, d)$. The universal set of bargaining solutions is denoted by $\mathcal{F}$.

To discuss explicitly the performances of bargaining solutions in resource allocation problems from a non-welfaristic point of view, we are not interested in the above defined, classical bargaining solution, but in its underlying resource allocation mechanism defined over the set of underlying economies. An allocation rule is a correspondence $\varphi: \mathcal{E} \rightarrow Z(\mathcal{E})$

\footnotetext{
${ }^{2}$ Such a definition of economic environments is originated from Roemer (1986).
} 
which associates to each $\mathbf{e}=(M, \mathbf{u}, \mathbf{s}, Y) \in \mathcal{E}$, a non-empty subset $\varphi(\mathbf{e})$ of $Z(\mathbf{e})$. The allocation rule $\varphi$ is assumed to be essentially a function; that is, for all $\mathbf{e}=(M, \mathbf{u}, \mathbf{s}, Y) \in$ $\mathcal{E}$, if $\mathbf{z} \in \varphi(\mathbf{e})$ and $\mathbf{z}^{\prime} \in \varphi(\mathbf{e})$, then $\mathbf{u}(\mathbf{z})=\mathbf{u}\left(\mathbf{z}^{\prime}\right)$, where $\mathbf{u}(\mathbf{z})=\left(u_{i}\left(z_{i}\right)\right)_{i \in N}$ and $\mathbf{u}\left(\mathbf{z}^{\prime}\right)=$ $\left(u_{i}\left(z_{i}^{\prime}\right)\right)_{i \in N}$. Moreover, $\varphi$ is assumed to be a full correspondence; that is, for all $\mathbf{e}=$ $(M, \mathbf{u}, \mathbf{s}, Y) \in \mathcal{E}$, if $\mathbf{z} \in \varphi(\mathbf{e}), \mathbf{z}^{\prime} \in Z(\mathbf{e})$, and $\mathbf{u}(\mathbf{z})=\mathbf{u}\left(\mathbf{z}^{\prime}\right)$, then $\mathbf{z}^{\prime} \in \varphi(\mathbf{e})$. The allocation rule $\varphi$ attains a bargaining solution $F$ if for all $\mathbf{e}=(M, \mathbf{u}, \mathbf{s}, Y) \in \mathcal{E}, \mu_{\varphi}(\mathbf{e})=F(S(\mathbf{e}))$, where $\mu_{\varphi}(\mathbf{e}) \equiv \mathbf{u}(\varphi(\mathbf{e}))$. Thus, $\varphi$ is the underlying resource allocation mechanism of the classical bargaining solution $F$ if and only if $\varphi$ attains $F$. Denote the class of all the allocation rules, each of which attains some bargaining solution, by $\Phi_{\mathcal{F}}$.

Among the various types of bargaining solutions, here we are particularly interested in the following ones:

Definition 1. A bargaining solution $N a \in \mathcal{F}$ is the Nash solution if for any $S \in \Sigma$, $N a(S)$ is equal to the maximizer in $\overline{\mathbf{u}} \in S$ of the "Nash product" $\prod_{i \in N} \bar{u}_{i}$.

Definition 2. An allocation rule $\varphi^{N a}$ is the Nash rule if it attains the Nash solution: for all $\mathbf{e} \in \mathcal{E}, \mu_{\varphi^{N a}}(\mathbf{e})=N a(S(\mathbf{e}))$.

Given $S \in \Sigma$ and $i \in N$, let us define $m^{i}(S) \equiv \max \left\{\bar{u}_{i} \in \mathbb{R}_{+} \mid \overline{\mathbf{u}}=\left(\bar{u}_{h}\right)_{h \in N} \in S\right\}$.

Definition 3. A bargaining solution $K \in \mathcal{F}$ is the Kalai-Smorodinsky solution if for any $S \in \Sigma, K(S)$ is a (weak) Pareto efficient outcome on $S$, and there exists a unique value $\lambda \in(0,1]$ such that $K(S)=\lambda \cdot \mathbf{m}(S), \mathbf{m}(S) \equiv\left(m^{i}(S)\right)_{i \in N}$.

Definition 4. An allocation rule $\varphi^{K}$ is the Kalai-Smorodinsky rule if it attains the Kalai-Smorodinsky solution: for all $\mathbf{e} \in \mathcal{E}, \mu_{\varphi^{K}}(\mathbf{e})=K(S(\mathbf{e}))$.

3. Axioms using economic information. First of all, the domain assumptions on $\varphi$ is:

Axiom $D^{\mathcal{E}}$ : The allocation rule $\varphi$ is a full correspondence which is essentially a function and is defined on the class of economies $\mathcal{E}$.

The following are well-known axioms on allocation rules:

Welfarism (W): For all $\mathbf{e}=(M, \mathbf{u}, \mathbf{s}, Y), \mathbf{e}^{\prime}=\left(M^{\prime}, \mathbf{u}^{\prime}, \mathbf{s}^{\prime}, Y^{\prime}\right) \in \mathcal{E}$, if $S(\mathbf{e})=S\left(\mathbf{e}^{\prime}\right)$, then $\mu_{\varphi}(\mathbf{e})=\mu_{\varphi}\left(\mathbf{e}^{\prime}\right)$.

Pareto Efficiency (PE): For all $\mathbf{e}=(M, \mathbf{u}, \mathbf{s}, Y) \in \mathcal{E}$ and $\mathbf{z} \in \varphi(\mathbf{e}), \mathbf{z}$ is a Pareto efficient allocation for $\mathbf{e}$.

Weak Equal Treatment of Equals (WETE): For all $\mathbf{e}=(M, \mathbf{u}, \mathbf{s}, Y) \in \mathcal{E}$, if $u_{i}=u_{j}$ and $s_{i}=s_{j}$ for all $i, j \in N$, then for all $\mathbf{z} \in \varphi(\mathbf{e}), u_{i}\left(z_{i}\right)=u_{j}\left(z_{j}\right)$ for all $i, j \in N$.

Note that all allocation rules in $\Phi_{\mathcal{F}}$ satisfy Axiom $D^{\mathcal{E}}$ and $\mathbf{W}$.

The next axiom was introduced by Yoshihara (2003) to stipulate the performance of allocation rules in the case of particular types of technological changes. It requires coherent treatments of allocation problems between before and after technological innovations if such innovations only make it possible to produce new commodities which nobody wants to consume: Given $(x, y) \in[0, \bar{x}] \times \mathbb{R}_{+}^{m}$ and $u_{i} \in \mathcal{U}^{M}$, let there be $K \subsetneq M$ such that for all $y_{K}^{\prime} \equiv\left(y_{f}^{\prime}\right)_{f \in K} \in \mathbb{R}_{+}^{k}, u_{i}\left(x, y_{K}^{\prime}, y_{M \backslash K}\right)=u_{i}\left(x, y_{K}, y_{M \backslash K}\right)$, where $y_{K} \equiv\left(y_{f}\right)_{f \in K}$. 
Then, we say that agent $i \in N$ is indifferent to each good of $K \subsetneq M$ at $(x, y)$. Given $Y \in \mathcal{Y}^{M}$ and $K \subsetneq M$, let

$$
P_{M \backslash K}(Y) \equiv\left\{\left(x, y_{M \backslash K}\right) \in \mathbb{R}_{+} \times \mathbb{R}_{+}^{m-k} \mid \exists y_{K} \in \mathbb{R}_{+}^{k}:\left(x, y_{K}, y_{M \backslash K}\right) \in Y\right\} .
$$

Consistency w.r.t. Technological Innovation (CTI): Let $\mathbf{e}=(M, \mathbf{u}, \mathbf{s}, Y) \in \mathcal{E}^{M}$, and let $\widehat{\mathbf{z}}=\left(\widehat{x}_{i}, \widehat{y}_{M i}\right)_{i \in N} \in \varphi(\mathbf{e})$ be weakly Pareto efficient. ${ }^{3}$ Let $\mathbf{e}^{\prime}=\left(M \cup L, \mathbf{u}^{\prime}, \mathbf{s}, Y^{\prime}\right) \in$ $\mathcal{E}^{M \cup L}$, where $M \cap L=\varnothing$, be such that (1) $P_{M}\left(Y^{\prime}\right)=Y$, (2) for any $\mathbf{z}=\left(x_{i}, y_{M i}\right)_{i \in N} \in$ $W P E(\mathbf{e})$, there exists $\left(y_{L i}(\mathbf{z})\right)_{i \in N} \in \mathbb{R}_{+}^{n l}$ such that

$$
u_{i}^{\prime}\left(x_{i}, y_{M i}, y_{L i}(\mathbf{z})\right)=u_{i}\left(x_{i}, y_{M i}\right)(\forall i \in N) \text { and }\left(x_{i}, y_{M i}, y_{L i}(\mathbf{z})\right)_{i \in N} \in W P E\left(\mathbf{e}^{\prime}\right),
$$

and (3) every agent $i \in N$ is indifferent to each good of $L$ at $\left(\widehat{x}_{i}, \widehat{y}_{M i}, \mathbf{0}\right)$. Then, $\left(\widehat{x}_{i}, \widehat{y}_{M i}, \mathbf{0}\right)_{i \in N} \in \varphi\left(\mathbf{e}^{\prime}\right)$.

A motivation for CTI is presented as follows: let, in an economy e with $M$-commodities, $\widehat{\mathbf{z}}=\left(\widehat{x}_{i}, \widehat{y}_{M i}\right)_{i \in N}$ be a recommendation by the allocation rule $\varphi$, and be a (weakly) Pareto efficient allocation. Next, let the economy change from $\mathbf{e} \in \mathcal{E}^{M}$ to $\mathbf{e}^{\prime} \in \mathcal{E}^{M \cup L}$, where the economy $\mathbf{e}^{\prime}$ inherits from e the characteristics of the agents' utility functions and production technology on $M$-commodities in the intimate way that CTI postulates. The main difference between $\mathbf{e}$ and $\mathbf{e}^{\prime}$ comes from the technological change from $Y$ to $Y^{\prime}$ which makes it possible to consume the new commodities $L$. On the contrary, it is a useless innovation because everyone's opportunity for welfare is not enlarged. Then, it may be reasonable that, in the new economy, every agent is guaranteed at least his/her original welfare level which is enjoyed by consuming $M$-commodities in the original economy. It follows from this view that $\left(\widehat{x}_{i}, \widehat{y}_{M i}, \mathbf{0}\right)_{i \in N}$ is a recommendation of $\varphi$ in $\mathbf{e}^{\prime}$. In fact, by this new recommendation, nothing is lost by individuals because of the environmental change since, in the new economy, no individuals want to consume $L$-commodities, and $\left(\widehat{x}_{i}, \widehat{y}_{M i}, \mathbf{0}\right)_{i \in N}$ is (weakly) Pareto efficient.

Remark 1. The axiom $\mathbf{W}$ implies CTI under Axiom $D^{\mathcal{E}}$, but not the converse, even if $\varphi$ obeys Axiom $D^{\mathcal{E}}$.

Since all allocation rules in $\Phi_{\mathcal{F}}$ satisfy $\mathbf{W}$ and Axiom $D^{\mathcal{E}}$, they also satisfy $\mathbf{C T I}$ by Remark 1.

3.1. Axioms on responsibility and compensation. In this section, we introduce axioms which are related to the arguments of responsibility and compensation.

3.1.1. Axiom on responsibility. The first axiom seems to be relevant to an individual's responsibility for his/her (cardinal) utility function. To define it, let us begin with introducing a few notions: Given $M \in \mathcal{M}$, note that for any utility function $u \in \mathcal{U}^{M}$, there is a utility-unit $b^{u} \in \mathbb{R}_{+}$, by which the level of utility assigned by the function $u$ is measured: that is, if $u(z)=b^{u}$ for some $z$, it implies that the level of utility $u(z)$ is just

\footnotetext{
${ }^{3}$ When we discuss dimensional changes in consumption spaces, we often denote individual $i^{\prime}$ s consumption vector of $M$-goods by $y_{M i} \in \mathbb{R}_{+}^{m}$.

${ }^{4}$ For more detailed discussion, see Yoshihara (2003).
} 
"one." ${ }^{5}$ Then, for each utility-unit $b^{u}$, there is a corresponding set $B(u) \subsetneq[0, \bar{x}] \times \mathbb{R}_{+}^{m}$ of base-consumption for $u$ such that for all $z \in B(u), u(z)=b^{u}$.

Now, let us take any two utility functions $u, u^{\prime} \in \mathcal{U}^{M}$ for which there is a positive scalar $\lambda>0$ such that $u^{\prime}=\lambda \cdot u$. If $\lambda=\frac{b^{u^{\prime}}}{b^{u}}$, then $u^{\prime}$ is just obtained by a change in utility-units from $b^{u}$ to $b^{u^{\prime}}$, so that $u$ and $u^{\prime}$ are essentially the same utility representation. In this case, note that $B(u)=B\left(u^{\prime}\right)$. In contrast, if $b^{u}=b^{u^{\prime}}$, then the change from $u$ to $u^{\prime}$ can be explained not by the change in utility-units, but rather by a change in utility intensity. Note that if the change from $u$ to $u^{\prime}$ comes from the change in utility intensity, then we have $B(u) \neq B\left(u^{\prime}\right)$ and $B(u) \cap B\left(u^{\prime}\right)=\varnothing .^{6}$

One typical example of the above change in utility intensity is the case of individual development of "expensive taste," which was discussed by Dworkin (1981a). Consider a case in which an individual develops his/her expensive taste, so that even if his/her underlying preference ordering and his/her risk attitude are invariant, he/she can no longer enjoy the same level of welfare as he/she did before developing his/her expensive taste, without receiving a larger consumption vector than before. ${ }^{7}$ This case is simply formulated as a process of a linear transformation of a utility function via a change in utility intensity.

Let us now define the first axiom. Given $M \in \mathcal{M}$ and $\mathbf{u} \in \mathcal{U}^{M n}$, let $\mathbf{b}^{\mathbf{u}} \equiv\left(b^{u_{i}}\right)_{i \in N}$. Then:

Independence of Utility Intensities (IUI) ${ }^{8}$ : For all $\mathbf{e}=(M, \mathbf{u}, \mathbf{s}, Y), \mathbf{e}^{\prime}=\left(M, \mathbf{u}^{\prime}, \mathbf{s}, Y\right)$ $\in \mathcal{E}$ with $\mathbf{b}^{\mathbf{u}}=\mathbf{b}^{\mathbf{u}^{\prime}}$, if there exists a vector $\mathbf{a}=\left(a_{i}\right)_{i \in N} \in \mathbb{R}_{++}^{n}$ such that $u_{i}^{\prime}=a_{i} \cdot u_{i}$ for all $i \in N$, then $\varphi(\mathbf{e})=\varphi\left(\mathbf{e}^{\prime}\right)$.

Our motivation for this responsibility-related axiom is presented as follows: in production economies with differences in production skills, but without differences in consumption abilities among agents, it seems to be that the change in utility intensity of any agent is not a subject for social compensation, but a matter of personal responsiblity. So, the allocation rule should not take into account such an environmental change in determining resource allocations. ${ }^{9}$

Note that Roemer (1988) introduced a similar axiom to IUI, say, Cardinal Noncomparability, which expresses exactly what Nash intended with his axiom of Scale In-

\footnotetext{
${ }^{5}$ The author owes the introduction of utility-units in defining the following two axioms to one of the referees of Yoshihara (2003).

${ }^{6}$ We can start from listing $B(u)$ instead of $b^{u}$ as primitive data. Then, by comparing $B(u)$ with $B\left(u^{\prime}\right)$, we can see which type of change occurs when $u$ and $u^{\prime}$ are correlated by a linear transformation: if $B(u)=B\left(u^{\prime}\right)$, it is a change in utility-units, while otherwise, it involves a change in utility intensity.

${ }^{7}$ In this explanation, it is not necessary to assume interpersonal comparability of utilities. The notion of change in utility intensity only presumes intrapersonal comparison of utilities.

${ }^{8}$ This axiom was first introduced by Yoshihara (2003).

${ }^{9}$ As discussed above, we may connect the situation that someone's utility intensity decreases with the development of an "expensive taste." Then, IUI requires that this person should not be compensated by the allocation rule for his decrease of utility-productivity due to his developed expensive taste.
} 
variance [Nash (1950)]. The motivation of Cardinal Non-comparability can be formulated in our model as follows:

Utility-units Invariance (UUI): For all $\mathbf{e}=(M, \mathbf{u}, \mathbf{s}, Y), \mathbf{e}^{\prime}=\left(M, \mathbf{u}^{\prime}, \mathbf{s}, Y\right) \in \mathcal{E}$ with $\mathbf{b}^{\mathbf{u}} \neq \mathbf{b}^{\mathbf{u}^{\prime}}$, if $u_{i}^{\prime}=\frac{b^{u_{i}^{\prime}}}{b^{u_{i}}} \cdot u_{i}$ for all $i \in N$, then $\varphi(\mathbf{e})=\varphi\left(\mathbf{e}^{\prime}\right)$.

Although both IUI and UUI are respectively implied by Nash's Scale Invariance axiom, their motivations are completely different from each other.

3.1.2. Axioms on compensation. The next two axioms are relevant to compensation for low sklls:

Skill Monotonicity $(\mathbf{S M})^{10}$ : For all $\mathbf{e}=(M, \mathbf{u}, \mathbf{s}, Y), \mathbf{e}^{\prime}=\left(M, \mathbf{u}, \mathbf{s}^{\prime}, Y\right) \in \mathcal{E}$ such that $\mathbf{s} \leq \mathbf{s}^{\prime}$, and all $\mathbf{z} \in \varphi(\mathbf{e})$ and $\mathbf{z}^{\prime} \in \varphi\left(\mathbf{e}^{\prime}\right), u_{i}\left(z_{i}\right) \leq u_{i}\left(z_{i}^{\prime}\right)$ for all $i \in N$.

Independence of Skill Endowments $(\mathrm{ISE})^{11}:$ Let $\mathbf{e}=(M, \mathbf{u}, \mathbf{s}, Y), \mathbf{e}^{\prime}=\left(M, \mathbf{u}, \mathbf{s}^{\prime}, Y\right)$ $\in \mathcal{E}$ be such that $Z(\mathbf{e})=Z\left(\mathbf{e}^{\prime}\right)$. Then, $\varphi(\mathbf{e})=\varphi\left(\mathbf{e}^{\prime}\right)$.

Among these two axioms, $\mathbf{S M}$ is strong enough to keep the implication of compensation for low skill, while ISE is a rather weak requirement as an axiom of compensation. In fact, since $\mathbf{W}$ implies ISE, every bargaining allocation rule in $\Phi_{\mathcal{F}}$ satisfies ISE. This implies, first, that all symmetric bargaining solutions are happy to have the property of compensating low skill agents, at least in the sense of ISE. It implies, secondly, that ISE is not such a powerful criterion for classifying bargaining solutions from the viewpoint of compensation for low skill. As we see below, there is no bargaining solution satisfying the responsibility axiom, IUI, and the stronger compensation axiom SM. Moreover, once we weaken the compensation requirement from SM to ISE, then the Nash [Nash (1950)] and the Kalai-Smorodinsky [Kalai and Smorodinsky (1975)] solutions satisfy both the requirements of responsibility and compensation [Yoshihara (2003)]. The last statement might not be so appealing, because ISE is a rather weak requirement as discussed above. So, we may need a new axiom of compensation, which may be stronger than ISE, but must be weaker than SM.

Thus, we now introduce a weaker version of SM, which is defined as follows:

$\alpha$-Weak Skill Monotonicity $(\alpha \mathbf{W S M}):$ Let $\mathbf{e}=(M, \mathbf{u}, \mathbf{s}, Y), \mathbf{e}^{\prime}=\left(M, \mathbf{u}, \mathbf{s}^{\prime}, Y\right) \in \mathcal{E}$ be such that $\mathbf{s} \leq \mathbf{s}^{\prime}$. Then, for all $\mathbf{z}^{\prime} \in \varphi\left(\mathbf{e}^{\prime}\right)$, if $\mathbf{z}^{\prime} \in P E(\mathbf{e})$, then, for all $\mathbf{z} \in \varphi(\mathbf{e})$, we have $u_{i}\left(z_{i}\right) \leq u_{i}\left(z_{i}^{\prime}\right)$ for all $i \in N$.

This axiom has two important implications. First, it is surely a weaker version of SM. Secondly, the axiom together with PE imply a solidarity condition for cases where someone happens to have a lower skill than the individual had before. This is because if $\mathbf{z}^{\prime} \in P E\left(\mathbf{e}^{\prime}\right)$, then $u_{i}\left(z_{i}\right)=u_{i}\left(z_{i}^{\prime}\right)$ must follow for all $\mathbf{z} \in \varphi(\mathbf{e})$ and for all $i \in N$. This implies the following situation: someone happens to have a lower skill, so that the environment changes from $\mathbf{e}^{\prime}$ to $\mathbf{e}$, but this worsening is not so serious that the allocation $\mathbf{z}^{\prime} \in \varphi\left(\mathbf{e}^{\prime}\right) \subseteq P E\left(\mathbf{e}^{\prime}\right)$ does not remain to be Pareto efficient for $\mathbf{e}$. Then, there is no reason

\footnotetext{
${ }^{10}$ This axiom was originated by Fleurbaey and Maniquet (1999).

${ }^{11}$ This axiom was first introduced by Yoshihara (2003).
} 
to make someone's welfare worse off in the new recommendation $\varphi(\mathbf{e})$, since, by $\mathbf{P E}$ of $\varphi$, such worsening of someone only implies that another's welfare increases.

Note that in axiomatic theories of the Nash bargaining solution [Nash (1950)], one of the most debatable issues has been the justification of Nash's independence of irrelevant alternatives (Nash IIA) axiom. Although Nash himself considered Nash IIA as a consistency condition which is an "institutional assumption" about the convention the players are to use in resolving bargaining games, it still remains unclear why Nash IIA is an appropriate consistency condition. Binmore (1987) replaced Nash IIA with his new axiom, Convention Consistency (CC), so that an asymmetric Nash bargaining solution was derived, although this new axiom entails essentially the same implication as Nash IIA. In contrast, $\alpha \mathbf{W S M}$ does not include the implication of convention consistency entailed in Nash IIA and CC.

We also introduce another weaker version of $\mathbf{S M}$, which is logically independent of $\alpha \mathbf{W S M}$ and defined as follows: Given $\mathbf{e}=(M, \mathbf{u}, \mathbf{s}, Y) \in \mathcal{E}$ and $i \in N$, let

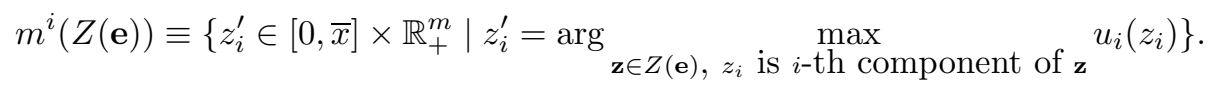

Then:

$\beta$-Weak Skill Monotonicity ( $\beta$ WSM): Let $\mathbf{e}=(M, \mathbf{u}, \mathbf{s}, Y), \mathbf{e}^{\prime}=\left(M, \mathbf{u}, \mathbf{s}^{\prime}, Y\right) \in \mathcal{E}$ be such that $\mathbf{s} \leq \mathbf{s}^{\prime}$, and $m^{i}(Z(\mathbf{e})) \cap m^{i}\left(Z\left(\mathbf{e}^{\prime}\right)\right) \neq \varnothing$ for all $i \in N$. Then, for all $\mathbf{z} \in \varphi(\mathbf{e})$ and all $\mathbf{z}^{\prime} \in \varphi\left(\mathbf{e}^{\prime}\right)$, we have $u_{i}\left(z_{i}\right) \leq u_{i}\left(z_{i}^{\prime}\right)$ for all $i \in N$.

This axiom requires that if the profile of skills happens to be improved, so that everyone's potential for welfare increases, but everyone's ideal utility point remains the same as before, then no individual should be made worse off by this environmental improvement. In what situations does everyone's ideal utility point remain the same as before, when the profile of skills is improved? Let $z_{i}$ and $z_{i}^{\prime}$ be $i^{\prime}$ s consumption vectors which belong to $m^{i}(Z(\mathbf{e}))$ and $m^{i}\left(Z\left(\mathbf{e}^{\prime}\right)\right)$ respectively. Without loss of generality, we can assume that $y_{i} \leq y_{i}^{\prime}$ and $x_{i}=x_{i}^{\prime}$, because $\mathbf{s} \leq \mathbf{s}^{\prime}$ for $\mathbf{e}=(M, \mathbf{u}, \mathbf{s}, Y), \mathbf{e}^{\prime}=\left(M, \mathbf{u}, \mathbf{s}^{\prime}, Y\right) \in \mathcal{E}$. However, if everyone's preference is satiated at $z_{i}$, then it could be that $m^{i}(Z(\mathbf{e})) \cap m^{i}\left(Z\left(\mathbf{e}^{\prime}\right)\right) \neq \varnothing$ for all $i \in N$. Note that such a situation does not necessarily remove the possibility of $S(\mathbf{e}) \subsetneq S\left(\mathbf{e}^{\prime}\right)$.

4. Main result. First of all, we should mention that there is no bargaining solution which satisfies both the responsibility and the stronger compensation requirements:

Proposition 1 [Yoshihara (2003)]. There is no allocation rule in $\Phi_{\mathcal{F}}$ which satisfies PE, WETE, IUI, and SM.

As one second best resolution, Yoshihara (2003) showed that we can remove the responsibility axiom IUI, while keeping the compensation axiom SM, which results in the egalitarian solution [Kalai (1977)]. As another second best resolution, we can replace the stronger compensation axiom SM with either (1) $\alpha \mathbf{W S M}$ or (2) $\beta \mathbf{W S M}$, while keeping the responsibility axiom IUI. In the way of (1), we can obtain the following result: 
Theorem 1. The allocation rule $\varphi$ satisfies $D^{\mathcal{E}}$, PE, WETE, ISE, $\alpha$ WSM, IUI, and CTI if and only if $\varphi=\varphi^{N a}$.

REMARK 2. It is also easy to see that the Nash rule $\varphi^{N a}$ is the unique allocation rule satisfying UUI and all the axioms in Theorem 1 except IUI.

Here, in contrast to the result of Yoshihara (2003), the Nash solution is characterized without the help of an informational efficiency axiom such as Independence of Technological Contraction (ITC) [Moulin (1990)]. ${ }^{1213}$ Instead, it is just characterized by the requirement of responsibility and a weak version of Skill Monotonicity. Moreover, since $D^{\mathcal{E}}$, ISE, and CTI are necessary conditions for all allocation rules in $\Phi_{\mathcal{F}}$, the above theorem implies the following proposition:

COROLlary 1. The Nash rule $\varphi^{N a}$ is the unique allocation rule in $\Phi_{\mathcal{F}}$ which satisfies PE, WETE, IUI, and $\alpha$ WSM.

Thus, Corollary 1 implies that the Nash solution is one type of second best, efficient, and symmetric solution, which satisfies both the responsibility and compensation requirements.

Next, we will discuss the replacement of (2). Let $\mathcal{E}^{M *} \subseteq \mathcal{E}^{M}$ be the class of economies with $M$-goods, whose utility possibility sets are strictly comprehensive. Let $\mathcal{E}^{*} \equiv$ $\bigcup_{M \in \mathcal{M}} \mathcal{E}^{M *}$. Then:

Axiom $D^{\mathcal{E}^{*}}$ : The allocation rule $\varphi$ is a full correspondence which is essentially a function and is defined on the class of economies $\mathcal{E}^{*}$.

Theorem 2. The allocation rule $\varphi$ satisfies $D^{\mathcal{E}^{*}}$, PE, WETE, ISE, $\beta$ WSM, IUI, and CTI if and only if $\varphi=\varphi^{K}$.

COROLlary 2. The Kalai-Smorodinsky rule $\varphi^{K}$ is the unique allocation rule in $\Phi_{\mathcal{F}}$ which satisfies PE, WETE, IUI, and $\beta \mathbf{W S M}$.

Since both $\alpha \mathbf{W S M}$ and $\beta$ WSM are weaker versions of SM, and they are logically independent from each other, ranking the Nash and the Kalai-Smorodinsky solutions on the basis of the responsibility and compensation view is not a trivial exercise. However, $\beta$ WSM seems not to be very appealing as a compensation axiom, since the premise of $\beta$ WSM has no particular ethical implication.

5. Proof of Theorem. Given $Y^{(m)} \in \mathcal{Y}^{M}$ and $Y^{(l)} \in \mathcal{Y}^{L}$ with $M \cap L=\varnothing$, let

$$
Y^{(m)} \oplus Y^{(l)} \equiv\left\{\left(x, y_{M}, y_{L}\right) \in \mathbb{R}_{+} \times \mathbb{R}_{+}^{m} \times \mathbb{R}_{+}^{l} \mid\left(x, y_{M}\right) \in Y^{(m)},\left(x, y_{L}\right) \in Y^{(l)}\right\} .
$$

The following four lemmas are used in Yoshihara (2003), all of which are also essential in this paper:

\footnotetext{
${ }^{12}$ Moulin (1990) introduced this axiom with the name of Nash IIA.

${ }^{13}$ Binmore (1987a) showed that in two-person exchange economies, the only solution satisfying all the Nash-like economic axioms he defined is the Walrasian solution. Since his result depends on a stronger domain restriction and a stronger economic version of Nash IIA than ITC, we cannot obtain the same relationship between the Nash and the Walrasian solutions in our economic domain.
} 
Lemma 1. Let $\mathbf{e}_{1}, \mathbf{e}_{2} \in \mathcal{E}$ be such that $\mathbf{e}_{1}=\left(M, \mathbf{u}^{1}, \mathbf{s}, Y^{(m)}\right) \in \mathcal{E}^{M}, \mathbf{e}_{2}=\left(L, \mathbf{u}^{2}, \mathbf{s}, Y^{(l)}\right) \in$ $\mathcal{E}^{L}$, and $S\left(\mathbf{e}_{1}\right)=S\left(\mathbf{e}_{2}\right)$. Then, the allocation rule $\varphi$ which satisfies $D^{\mathcal{E}}, \mathbf{P E}$, and $\mathbf{C T I}$ has the following property: $\mu_{\varphi}\left(\mathbf{e}_{1}\right)=\mu_{\varphi}\left(\mathbf{e}_{2}\right)$.

Lemma 2. Let $\mathbf{e}_{1}, \mathbf{e}_{2} \in \mathcal{E}$ be such that $\mathbf{e}_{1}=\left(M, \mathbf{u}^{1}, \mathbf{s}, Y^{(m)}\right) \in \mathcal{E}^{M}$ and $\mathbf{e}_{2}=\left(L, \mathbf{u}^{2}, \mathbf{s}, Y^{(l)}\right)$ $\in \mathcal{E}^{L}$ with $\mathbf{b}^{\mathbf{u}^{1}}=\mathbf{b}^{\mathbf{u}^{2}}$. Moreover, there exists $\mathbf{a}=\left(a_{i}\right)_{i \in N} \in \mathbb{R}_{+}^{n}$ such that

$$
\overline{\mathbf{u}}=\left(\bar{u}_{i}\right)_{i \in N} \in S\left(\mathbf{e}_{1}\right) \Leftrightarrow \mathbf{a} \cdot \overline{\mathbf{u}}=\left(a_{i} \cdot \bar{u}_{i}\right)_{i \in N} \in S\left(\mathbf{e}_{2}\right) .
$$

Then, if the allocation rule $\varphi$ satisfies $D^{\mathcal{E}}$, PE, IUI, and CTI, then $\mu_{\varphi}\left(\mathbf{e}_{2}\right)=\mathbf{a} \cdot \mu_{\varphi}\left(\mathbf{e}_{1}\right)$.

Lemma 3. Let $\mathbf{e}=\left(M, \mathbf{u}, \mathbf{s}, Y^{(m)}\right) \in \mathcal{E}$ be such that $S(\mathbf{e})$ is a symmetric utility possibility set. Then, if the allocation rule $\varphi$ satisfies $D^{\mathcal{E}}, \mathbf{P E}, \mathbf{W E T E}$, ISE, and CTI, then $\mu_{\varphi i}(\mathbf{e})=\mu_{\varphi j}(\mathbf{e})$ for all $i, j \in N$.

Lemma 4. If $\varphi$ satisfies $D^{\mathcal{E}}$, PE, ISE, and CTI, then $\varphi$ satisfies $\mathbf{W}$.

The next lemma is essential to derive the Nash solution:

Lemma 5. Let $\mathbf{e}_{1}, \mathbf{e}_{2} \in \mathcal{E}$ be such that $\mathbf{e}_{1}=\left(M, \mathbf{u}^{1}, \mathbf{s}, Y^{(m)}\right) \in \mathcal{E}^{M}$ and $\mathbf{e}_{2}=\left(L, \mathbf{u}^{2}, \mathbf{s}, Y^{(l)}\right)$ $\in \mathcal{E}^{L}$. Moreover, $S\left(\mathbf{e}_{1}\right) \supseteq S\left(\mathbf{e}_{2}\right)$ with $\mu_{\varphi}\left(\mathbf{e}_{1}\right) \in S\left(\mathbf{e}_{2}\right)$ holds. Then, if the allocation rule $\varphi$ satisfies $D^{\mathcal{E}}, \mathbf{P E}, \alpha \mathbf{W S M}$, and $\mathbf{C T I}$, then $\mu_{\varphi}\left(\mathbf{e}_{1}\right)=\mu_{\varphi}\left(\mathbf{e}_{2}\right)$.

Proof. 1. Given $\mathbf{e}_{1}=\left(M, \mathbf{u}^{1}, \mathbf{s}, Y^{(m)}\right) \in \mathcal{E}^{M}$ and $\mathbf{e}_{2}=\left(L, \mathbf{u}^{2}, \mathbf{s}, Y^{(l)}\right) \in \mathcal{E}^{L}$ such that $S\left(\mathbf{e}_{1}\right) \supseteq S\left(\mathbf{e}_{2}\right)$, there exist other economies $\mathbf{e}_{1}^{\triangle}=\left(K, \mathbf{v}^{1}, \mathbf{s}, Y^{(\# K)}\right) \in \mathcal{E}^{K}$ and $\mathbf{e}_{2}^{\triangle}=$ $\left(K^{\prime}, \mathbf{v}^{2}, \mathbf{s}, Y^{\left(\# K^{\prime}\right)}\right) \in \mathcal{E}^{K^{\prime}}$ such that $K \cap K^{\prime}=\varnothing, S\left(\mathbf{e}_{1}^{\triangle}\right)=S\left(\mathbf{e}_{1}\right)$, and $S\left(\mathbf{e}_{2}^{\triangle}\right)=S\left(\mathbf{e}_{2}\right)$, which are guaranteed by Billera and Bixby (1973). Note that $Y^{(\# K)} \equiv \mathbb{R}_{+} \times[0,1]^{\# K}$ with generic element $\left(x, y_{K}\right) \in Y^{(\# K)}$ and $Y^{\left(\# K^{\prime}\right)} \equiv \mathbb{R}_{+} \times[0,1]^{\# K^{\prime}}$ with generic element $\left(x, y_{K^{\prime}}\right) \in Y^{\left(\# K^{\prime}\right)}$. Moreover, for each $i \in N$, the utility function $v_{i}^{1}:[0, \bar{x}] \times \mathbb{R}_{+}^{\# K} \rightarrow \mathbb{R}_{+}$ is defined as:

$$
\forall\left(x, y_{K}\right) \in[0, \bar{x}] \times \mathbb{R}_{+}^{\# K}, \quad v_{i}^{1}\left(x, y_{K}\right)= \begin{cases}v_{i}\left(y_{K}\right) & \text { if } y_{K} \in[0,1]^{\# K}, \\ v_{i}\left(\left(\min \left\{y_{f}, 1\right\}\right)_{f \in K}\right) & \text { otherwise, }\end{cases}
$$

and the utility function $v_{i}^{2}:[0, \bar{x}] \times \mathbb{R}_{+}^{\# K^{\prime}} \rightarrow \mathbb{R}_{+}$is defined as:

$$
\forall\left(x, y_{K^{\prime}}\right) \in[0, \bar{x}] \times \mathbb{R}_{+}^{\# K^{\prime}}, v_{i}^{2}\left(x, y_{K^{\prime}}\right)= \begin{cases}v_{i}^{\prime}\left(y_{K^{\prime}}\right) & \text { if } y_{K^{\prime}} \in[0,1]^{\# K^{\prime}}, \\ v_{i}^{\prime}\left(\left(\min \left\{y_{f^{\prime}}, 1\right\}\right)_{f^{\prime} \in K^{\prime}}\right) & \text { otherwise, }\end{cases}
$$

where the existence of concave, continuous, and monotonic utility functions $v_{i}:[0,1]^{\# K}$ $\rightarrow \mathbb{R}_{+}$and $v_{i}^{\prime}:[0,1]^{\# K^{\prime}} \rightarrow \mathbb{R}_{+}$with $v_{i}(\mathbf{0})=0$ and $v_{i}^{\prime}(\mathbf{0})=0$ are guaranteed by Billera and Bixby (1973). Construct the convolution $\mathbf{e}^{*}=\mathbf{e}_{1}^{\triangle} \wedge \mathbf{e}_{2}^{\triangle}=\left(K \cup K^{\prime}, \mathbf{v}^{*}, \mathbf{s}, Y^{(\# K)} \oplus\right.$ $\left.Y^{\left(\# K^{\prime}\right)}\right) \in \mathcal{E}^{K \cup K^{\prime}}$, where for all $i \in N, v_{i}^{*}\left(x, y_{K}, y_{K^{\prime}}\right)=\min \left\{v_{i}^{1}\left(x, y_{K}\right), v_{i}^{2}\left(x, y_{K^{\prime}}\right)\right\}$. In the same way as in step 2.1 of the proof of Lemma 2 in [Yoshihara (2003)], we can show that $S\left(\mathbf{e}^{*}\right)=S\left(\mathbf{e}_{1}^{\triangle}\right) \cap S\left(\mathbf{e}_{2}^{\triangle}\right)=S\left(\mathbf{e}_{2}\right)$. Thus, $\mu_{\varphi}\left(\mathbf{e}^{*}\right)=\mu_{\varphi}\left(\mathbf{e}_{2}\right)$ by Lemma 1 in this paper.

2. Construct the flat extension economy of $\mathbf{e}_{1}^{\triangle}$ :

$$
\widehat{\mathbf{e}}_{1}^{\triangle}=\left(K \cup K^{\prime}, \widehat{\mathbf{v}}^{1}, \mathbf{s}, Y^{(\# K)} \oplus Y^{\left(\# K^{\prime}\right)}\right)
$$

with $\widehat{v}_{i}^{1}\left(x, y_{K}, y_{K^{\prime}}\right)=v_{i}^{1}\left(x, y_{K}\right), \forall\left(x, y_{K}, y_{K^{\prime}}\right) \in[0, \bar{x}] \times \mathbb{R}_{+}^{\# K} \times \mathbb{R}_{+}^{\# K^{\prime}}$.

Since $S\left(\mathbf{e}_{1}\right)=S\left(\widehat{\mathbf{e}}_{1}^{\triangle}\right), \mu_{\varphi}\left(\mathbf{e}_{1}\right)=\mu_{\varphi}\left(\widehat{\mathbf{e}}_{1}^{\triangle}\right)$ by Lemma 1. Compare $\mathbf{e}^{*}$ with $\widehat{\mathbf{e}}_{1}^{\triangle}$. By definition, $\widehat{v}_{i}^{1} \geq v_{i}^{*}$ for all $i \in N$. 
3. By applying the Howe theorem [Howe (1987; Proposition 3, p. 59)], for each $i \in N$, there exist $w_{i} \in \mathcal{U}^{K \cup K^{\prime} \cup\{R(i)\}}$ and $\widehat{y}_{R(i)} \in \mathbb{R}_{+}$such that, for all $\left(x, y_{K}, y_{K^{\prime}}\right) \in[0, \bar{x}] \times$ $\mathbb{R}_{+}^{\# K} \times \mathbb{R}_{+}^{\# K^{\prime}}$

$$
w_{i}\left(x, y_{K}, y_{K^{\prime}}, \widehat{y}_{R(i)}\right)=\widehat{v}_{i}^{1}\left(x, y_{K}, y_{K^{\prime}}\right) \text { and } w_{i}\left(x, y_{K}, y_{K^{\prime}}, 0\right)=v_{i}^{*}\left(x, y_{K}, y_{K^{\prime}}\right) \text {. }
$$

Let $\mathbf{s}^{1}=\left(s_{i}^{1}\right)_{i \in N}$ be a new profile of production skills such that for all $i \in N$, $s_{i}^{1}=s^{1} \equiv 1$. Also, let $\mathbf{s}^{*}=\left(s_{i}^{*}\right)_{i \in N}$ be a new profile of production skills such that for all $i \in N, s_{i}^{*}=0$. Let, for each $R(i)$,

$$
Y^{R(i)} \equiv\left\{\left(x, y_{R(i)}\right) \in \mathbb{R}_{+}^{2} \mid y_{R(i)} \leq \min \left\{\frac{\widehat{y}_{R(i)}}{n \bar{x}} x, \widehat{y}_{R(i)}\right\}\right\} \text {. }
$$

Now, define

$$
\begin{aligned}
\widetilde{\widehat{\mathbf{e}}}_{1}^{\triangle} & \equiv\left(K \cup K^{\prime} \cup R, \widehat{\mathbf{w}}, \mathbf{s}^{1}, Y^{(\# K)} \oplus Y^{\left(\# K^{\prime}\right)} \oplus Y^{R}\right), \\
\widetilde{\mathbf{e}}^{*} & \equiv\left(K \cup K^{\prime} \cup R, \widehat{\mathbf{w}}, \mathbf{s}^{*}, Y^{(\# K)} \oplus Y^{\left(\# K^{\prime}\right)} \oplus Y^{R}\right), \\
\text { where } \quad Y^{R} & \equiv Y^{R(1)} \oplus \cdots \oplus Y^{R(i)} \oplus \cdots \oplus Y^{R(n)},
\end{aligned}
$$

and, for each $i \in N$,

$$
\begin{aligned}
& \widehat{w}_{i}\left(x, y_{K}, y_{K^{\prime}}, y_{R}\right)=w_{i}\left(x, y_{K}, y_{K^{\prime}}, y_{R(i)}\right) \\
& \qquad \text { for all }\left(x, y_{K}, y_{K^{\prime}}, y_{R}\right) \in[0, \bar{x}] \times \mathbb{R}_{+}^{\# K} \times \mathbb{R}_{+}^{\# K^{\prime}} \times \mathbb{R}_{+}^{n},
\end{aligned}
$$

where $R \equiv\{R(i)\}_{i \in N}$ and $y_{R}=\left(y_{R(i)}\right)_{i \in N}$. By Lemma 4, $\mu_{\varphi}\left(\widetilde{\widehat{\mathbf{e}}}_{1}^{\triangle}\right)=\mu_{\varphi}\left(\mathbf{e}_{1}\right)$ and $\mu_{\varphi}\left(\widetilde{\mathbf{e}}^{*}\right)=\mu_{\varphi}\left(\mathbf{e}_{2}\right)$.

4. Since $S\left(\widehat{\mathbf{e}}_{1}^{\triangle}\right)=S\left(\widetilde{\widehat{\mathbf{e}}}_{1}^{\triangle}\right)$, there exists $\left(\widehat{x}_{i}, \widehat{y}_{K i}, \widehat{y}_{K^{\prime} i}\right)_{i \in N} \in \varphi\left(\widehat{\mathbf{e}}_{1}^{\triangle}\right)$ such that $\left(\widehat{v}_{i}^{1}\left(\widehat{x}_{i}, \widehat{y}_{K i}, \widehat{y}_{K^{\prime} i}\right)\right)_{i \in N}=\left(\widehat{w}_{i}\left(\widehat{x}_{i}, \widehat{y}_{K i}, \widehat{y}_{K^{\prime} i}, \widehat{y}_{R(i)}, \mathbf{0}\right)\right)_{i \in N}=\mu_{\varphi}\left(\widetilde{\widehat{\mathbf{e}}}_{1}^{\triangle}\right)$. Since $\mu_{\varphi}\left(\mathbf{e}_{1}\right)=$ $\mu_{\varphi}\left(\widehat{\mathbf{e}}_{1}^{\triangle}\right) \in S\left(\mathbf{e}_{2}\right)=S\left(\widetilde{\mathbf{e}}^{*}\right)$, there exists $\zeta=\left(\widehat{x}_{i}^{\prime}, \widehat{y}_{K i}^{\prime}, \widehat{y}_{K^{\prime} i}^{\prime}, \mathbf{0}\right)_{i \in N} \in Z\left(\widetilde{\mathbf{e}}^{*}\right)$ such that $\left(\widehat{w}_{i}\left(\zeta_{i}\right)\right)_{i \in N}=\mu_{\varphi}\left(\mathbf{e}_{1}\right)=\mu_{\varphi}\left(\widetilde{\widehat{\mathbf{e}}}_{1}^{\triangle}\right)$. But, $\zeta$ is also a feasible allocation in $\widetilde{\widehat{\mathbf{e}}}_{1}^{\triangle}$. Moreover, $\zeta$ is Pareto efficient in $\widetilde{\widehat{\mathbf{e}}}_{1}^{\triangle}$ as well as in $\widetilde{\mathbf{e}}^{*}$, since $\mu_{\varphi}\left(\widetilde{\widehat{\mathbf{e}}}_{1}^{\triangle}\right)$ is a Pareto efficient utility allocation and $\mu_{\varphi}\left(\widetilde{\widehat{\mathbf{e}}}_{1}^{\triangle}\right) \in S\left(\widetilde{\mathbf{e}}^{*}\right) \subseteq S\left(\widetilde{\widehat{\mathbf{e}}}_{1}^{\triangle}\right)$. Since $\varphi$ is a full correspondence, $\zeta \in \varphi\left(\widetilde{\widehat{\mathbf{e}}}_{1}^{\triangle}\right)$. By $\alpha \mathbf{W S M}$ and PE, $\zeta \in \varphi\left(\widetilde{\mathbf{e}}^{*}\right)$. Thus, $\left(\widehat{w}_{i}\left(\zeta_{i}\right)\right)_{i \in N}=\mu_{\varphi}\left(\widetilde{\mathbf{e}}^{*}\right)=\mu_{\varphi}\left(\mathbf{e}_{2}\right)$ by Lemma 4 . This implies $\mu_{\varphi}\left(\mathbf{e}_{1}\right)=\mu_{\varphi}\left(\mathbf{e}_{2}\right)$.

Given $\mathbf{s} \in \mathcal{S}^{n}$, let $\mathcal{E}(\mathbf{s}) \subsetneq \mathcal{E}$ be the class of economies with the profile of production skills $\mathbf{s}$ fixed. Let $\left\{\mathcal{U}^{n}\left(\mathbf{b}_{\lambda}\right)\right\}_{\lambda \in \Lambda}$ be a partition of $\mathcal{U}^{n}$ such that for every $\lambda \in \Lambda$, every $n$-tuple utility functions in $\mathcal{U}^{n}\left(\mathbf{b}_{\lambda}\right)$ has the same profile of utility-units $\mathbf{b}_{\lambda}$. Given $\mathbf{s} \in \mathcal{S}^{n}$, let $\mathcal{E}\left(\mathbf{s} ; \mathbf{b}_{\lambda}\right) \subsetneq \mathcal{E}(\mathbf{s})$ be the class of economies with the profiles of production skills $\mathbf{s}$ and of utility-units $\mathbf{b}_{\lambda}$ fixed.

Proof of Theorem 1. (1) It is easy to see that $\varphi^{N a}$ satisfies $D^{\mathcal{E}}$, PE, WETE, ISE, $\alpha$ WSM, IUI, and CTI.

(2) Suppose that the allocation rule $\varphi$ satisfies $D^{\mathcal{E}}$, PE, WETE, ISE, $\alpha$ WSM, IUI, and CTI. Then, for any $\mathbf{s} \in \mathcal{S}^{n}$ and any $\mathbf{b}_{\lambda}, \mu_{\varphi}$ satisfies, on $\mathcal{E}\left(\mathbf{s} ; \mathbf{b}_{\lambda}\right)$, all four axioms which together characterize the Nash solution $N a$ [Nash (1950)], which is followed by Lemmas 
2, 3, and 5. Thus, for any $\mathbf{s} \in \mathcal{S}^{n}$ and any $\mathbf{b}_{\lambda}, \mu_{\varphi}$ on $\mathcal{E}\left(\mathbf{s} ; \mathbf{b}_{\lambda}\right)$ is always the outcome of the Nash solution $N a$. This implies that $\varphi$ attains $N a$, so that $\varphi=\varphi^{N a}$.

LEMma 6. Let $\mathbf{e}_{1}, \mathbf{e}_{2} \in \mathcal{E}^{*}$ be such that $\mathbf{e}_{1}=\left(M, \mathbf{u}^{1}, \mathbf{s}, Y^{(m)}\right) \in \mathcal{E}^{M *}$ and $\mathbf{e}_{2}=$ $\left(L, \mathbf{u}^{2}, \mathbf{s}, Y^{(l)}\right) \in \mathcal{E}^{L *}$. Moreover, $S\left(\mathbf{e}_{1}\right) \supseteq S\left(\mathbf{e}_{2}\right)$, and for all $i \in N, m^{i}\left(S\left(\mathbf{e}_{1}\right)\right)=$ $m^{i}\left(S\left(\mathbf{e}_{2}\right)\right)$. Then, if the allocation rule $\varphi$ satisfies $D^{\mathcal{E}^{*}}, \mathbf{P E}, \beta \mathbf{W S M}$, and $\mathbf{C T I}$, then $\mu_{\varphi}\left(\mathbf{e}_{1}\right) \geq \mu_{\varphi}\left(\mathbf{e}_{2}\right)$.

Proof. 1. Given $\mathbf{e}_{1}=\left(M, \mathbf{u}^{1}, \mathbf{s}, Y^{(m)}\right) \in \mathcal{E}^{M *}$ and $\mathbf{e}_{2}=\left(L, \mathbf{u}^{2}, \mathbf{s}, Y^{(l)}\right) \in \mathcal{E}^{L *}$ such that $S\left(\mathbf{e}_{1}\right) \supseteq S\left(\mathbf{e}_{2}\right)$, let us construct $\mathbf{e}_{1}^{\triangle}=\left(K, \mathbf{v}^{1}, \mathbf{s}, Y^{(\# K)}\right) \in \mathcal{E}^{K *}$ and $\mathbf{e}_{2}^{\triangle}=\left(K^{\prime}, \mathbf{v}^{2}, \mathbf{s}, Y^{\left(\# K^{\prime}\right)}\right)$ $\in \mathcal{E}^{K^{\prime} *}$ such that $K \cap K^{\prime}=\varnothing, S\left(\mathbf{e}_{1}^{\triangle}\right)=S\left(\mathbf{e}_{1}\right)$, and $S\left(\mathbf{e}_{2}^{\triangle}\right)=S\left(\mathbf{e}_{2}\right)$, as in step 5.1 of the proof of Lemma 5 . In the same way as in step 2.1 of the proof of Lemma 2 in [Yoshihara (2003)], we can show that $S\left(\mathbf{e}^{*}\right)=S\left(\mathbf{e}_{1}^{\triangle}\right) \cap S\left(\mathbf{e}_{2}^{\triangle}\right)=S\left(\mathbf{e}_{2}\right)$. Thus, $\mu_{\varphi}\left(\mathbf{e}^{*}\right)=\mu_{\varphi}\left(\mathbf{e}_{2}\right)$ by Lemma 1 in this paper.

2. Construct the flat extension economy of $\mathbf{e}_{1}^{\triangle}$ :

$$
\widehat{\mathbf{e}}_{1}^{\triangle}=\left(K \cup K^{\prime}, \widehat{\mathbf{v}}^{1}, \mathbf{s}, Y^{(\# K)} \oplus Y^{\left(\# K^{\prime}\right)}\right)
$$

with $\widehat{v}_{i}^{1}\left(x, y_{K}, y_{K^{\prime}}\right)=v_{i}^{1}\left(x, y_{K}\right), \forall\left(x, y_{K}, y_{K^{\prime}}\right) \in[0, \bar{x}] \times \mathbb{R}_{+}^{\# K} \times \mathbb{R}_{+}^{\# K^{\prime}}$.

Since $S\left(\mathbf{e}_{1}\right)=S\left(\widehat{\mathbf{e}}_{1}^{\triangle}\right), \mu_{\varphi}\left(\mathbf{e}_{1}\right)=\mu_{\varphi}\left(\widehat{\mathbf{e}}_{1}^{\triangle}\right)$ by Lemma 1. Compare $\mathbf{e}^{*}$ with $\widehat{\mathbf{e}}_{1}^{\triangle}$. By definition, $\widehat{v}_{i}^{1} \geq v_{i}^{*}$ for all $i \in N$. Since $m^{i}\left(S\left(\mathbf{e}_{1}\right)\right)=m^{i}\left(S\left(\mathbf{e}_{2}\right)\right)$ for all $i \in N$ and $S\left(\mathbf{e}_{1}\right)=S\left(\widehat{\mathbf{e}}_{1}^{\triangle}\right) \supseteq$ $S\left(\mathbf{e}^{*}\right)=S\left(\mathbf{e}_{2}\right)$, we obtain $m^{i}\left(S\left(\widehat{\mathbf{e}}_{1}^{\triangle}\right)\right)=m^{i}\left(S\left(\mathbf{e}^{*}\right)\right)$ for all $i \in N$.

3. By applying the Howe theorem [Howe (1987; Proposition 3, p. 59)], for each $i \in N$, there exist $w_{i} \in \mathcal{U}^{K \cup K^{\prime} \cup\{R(i)\}}$ and $\widehat{y}_{R(i)} \in \mathbb{R}_{+}$such that, for all $\left(x, y_{K}, y_{K^{\prime}}\right) \in[0, \bar{x}] \times$ $\mathbb{R}_{+}^{\# K} \times \mathbb{R}_{+}^{\# K^{\prime}}$

$$
w_{i}\left(x, y_{K}, y_{K^{\prime}}, \widehat{y}_{R(i)}\right)=\widehat{v}_{i}^{1}\left(x, y_{K}, y_{K^{\prime}}\right) \text { and } w_{i}\left(x, y_{K}, y_{K^{\prime}}, 0\right)=v_{i}^{*}\left(x, y_{K}, y_{K^{\prime}}\right) \text {. }
$$

Let $\mathbf{s}^{1}=\left(s_{i}^{1}\right)_{i \in N}$ and $\mathbf{s}^{*}=\left(s_{i}^{*}\right)_{i \in N}$ be respectively new profiles of production skills, which are defined exactly as in step 5.3 of the proof of Lemma 5 . Let, for each $R(i)$, define $Y^{R(i)}$ exactly as in step 5.3 of the proof of Lemma 5 . Now, define $\widetilde{\widehat{\mathbf{e}}}_{1}^{\triangle}, \widetilde{\mathbf{e}}^{*} \in \mathcal{E}^{K \cup K^{\prime} \cup R *}$ exactly as in step 5.3 of the proof of Lemma 5 . Then, $S\left(\widehat{\mathbf{e}}_{1}^{\triangle}\right)=S\left(\widetilde{\widehat{\mathbf{e}}}_{1}^{\triangle}\right) \supseteq S\left(\widetilde{\mathbf{e}}^{*}\right)=S\left(\mathbf{e}^{*}\right)$. 4. Since $m^{i}\left(S\left(\widehat{\mathbf{e}}_{1}^{\triangle}\right)\right)=m^{i}\left(S\left(\widetilde{\widehat{\mathbf{e}}}_{1}^{\triangle}\right)\right)$ and $m^{i}\left(S\left(\widetilde{\mathbf{e}}^{*}\right)\right)=m^{i}\left(S\left(\mathbf{e}^{*}\right)\right)$ for all $i \in N$, we have $m^{i}\left(S\left(\widetilde{\widehat{\mathbf{e}}}_{1}^{\triangle}\right)\right)=m^{i}\left(S\left(\widetilde{\mathbf{e}}^{*}\right)\right)$ for all $i \in N$. This implies that $m^{i}\left(Z\left(\widetilde{\widehat{\mathbf{e}}}_{1}^{\triangle}\right)\right) \cap m^{i}\left(Z\left(\widetilde{\mathbf{e}}^{*}\right)\right) \neq \varnothing$ for all $i \in N$, since $\mathbf{s}^{1} \geq \mathbf{s}^{*}$. Then, by $\beta \mathbf{W S M}, \mu_{\varphi}\left(\widetilde{\widehat{\mathbf{e}}}_{1}^{\triangle}\right) \geq \mu_{\varphi}\left(\widetilde{\mathbf{e}}^{*}\right)$. Thus, by Lemma 4 , $\mu_{\varphi}\left(\mathbf{e}_{1}\right)=\mu_{\varphi}\left(\widehat{\mathbf{e}}_{1}^{\triangle}\right)=\mu_{\varphi}\left(\widetilde{\widehat{\mathbf{e}}}_{1}^{\triangle}\right) \geq \mu_{\varphi}\left(\widetilde{\mathbf{e}}^{*}\right)=\mu_{\varphi}\left(\mathbf{e}^{*}\right)=\mu_{\varphi}\left(\mathbf{e}_{2}\right)$ holds.

Proof of Theorem 2. (1) It is easy to see that $\varphi^{K}$ satisfies $D^{\mathcal{E}^{*}}$, PE, WETE, ISE, $\beta \mathbf{W S M}$, IUI, and CTI.

(2) Suppose that the allocation rule $\varphi$ satisfies $D^{\mathcal{E}^{*}}$, PE, WETE, ISE, $\beta$ WSM, IUI, and CTI. Then, for any $\mathbf{s} \in \mathcal{S}^{n}$ and any $\mathbf{b}_{\lambda}, \mu_{\varphi}$ satisfies, on $\mathcal{E}^{*}\left(\mathbf{s} ; \mathbf{b}_{\lambda}\right)$, all four axioms which together characterize the Kalai-Smorodinsky solution $K$ [Thomson (1980)], which is followed by Lemmas 2,3 , and 6 . Thus, for any $\mathbf{s} \in \mathcal{S}^{n}$ and any $\mathbf{b}_{\lambda}, \mu_{\varphi}$ on $\mathcal{E}^{*}\left(\mathbf{s} ; \mathbf{b}_{\lambda}\right)$ is always the outcome of the Kalai-Smorodinsky solution $K$. This implies that $\varphi$ attains $K$, so that $\varphi=\varphi^{K}$. 


\section{References}

L. Billea and R. Bixby (1973), A characterization of Pareto surfaces, Proceedings of the American Mathematical Society 41, 261-267.

K. Binmore (1987), Nash bargaining theory I, in: The Economics of Bargaining, K. Binmore and P. Dasgupta (eds.), Blackwell, Oxford, 27-46.

K. Binmore (1987a), Nash bargaining theory III, in: The Economics of Bargaining, K. Binmore and P. Dasgupta (eds.), Blackwell, Oxford, 239-256.

R. Dworkin (1981a), What is equality? Part 1: Equality of welfare, Philosophy and Public Affairs $10,185-246$.

R. Dworkin (1981b), What is equality? Part 2: Equality of resources, Philosophy and Public Affairs 10, 283-345.

M. Fleurbaey and F. Maniquet (1996), Fair allocation with unequal production skills: the no-envy approach to compensation, Mathematical Social Sciences 32, 71-93.

M. Fleurbaey and F. Maniquet (1999). Fair allocation with unequal production skills: the solidarity approach to compensation, Social Choice and Welfare 16, 569-584.

R. Howe (1987), Sections and extensions of concave functions, Journal of Mathematical Economics 16, 53-64.

E. Kalai (1977), Proportional solutions to bargaining situations: interpersonal utility comparisons, Econometirca 45, 1623-1630.

E. Kalai and M. Smorodinsky (1975), Other solutions to Nash's bargaining problem, Econometirca 43, 513-518.

H. Moulin (1990), Joint ownership of a convex technology: comparison of three solutions, Review of Economic Studies 57, 439-452.

J. E. Roemer (1986), Equality of resources implies equality of welfare, Quarterly Journal of Economics 101, 751-784.

J. E. Roemer (1988), Axiomatic bargaining theory on economic environments, Journal of Economic Theory 45, 1-31.

W. Thomson (1980), Two characterizations of the Raiffa solution, Economics Letters 6, 225-231.

N. Yoshihara (2003), Characterizations of bargaining solutions in production economies with unequal skills, Journal of Economic Theory 108, 256-285. 